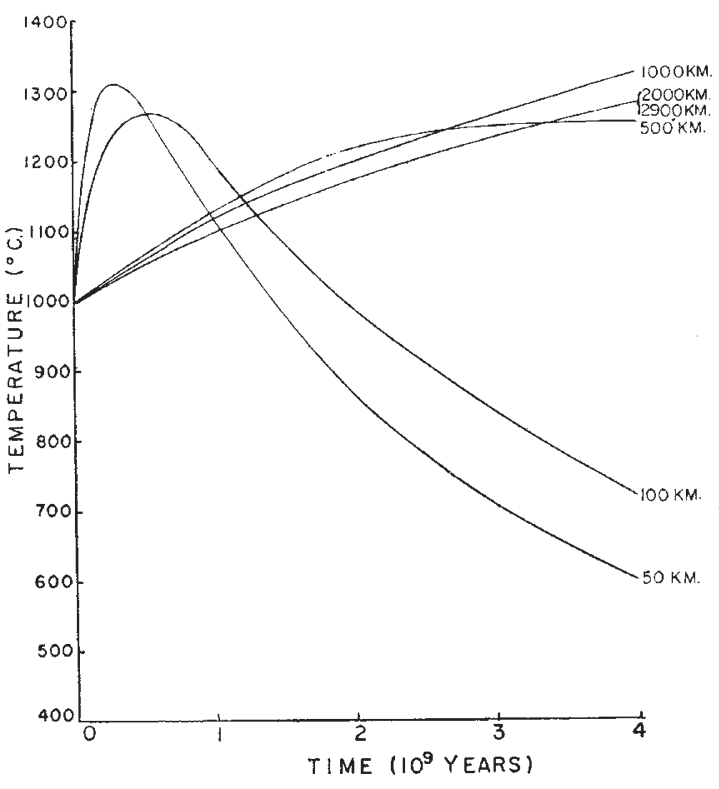

Fig. 5. Temperature-time distribution at various depths within the Earth (Model IV $B$ )

with time, and perhaps have been caused by different processes in the far past. The present temperatures at depths of $50-100 \mathrm{~km}$. differ by as much as $200^{\circ} \mathrm{C}$. between Models III $A$ and III $B$. These models are representative of conditions beneath the oceans and continents, and such a temperature difference may well exist and have an important bearing on oro- genesis. Fig. 5 gives the temperature distribution for Model IV $B$-an Earth with a cold origin. Here the thermal history is very dependent on the value assumed for the constant initial temperature. As shown in Fig. 5, if this is taken as $1,000^{\circ} \mathrm{C}$., there is heating at depth and an initial temperature rise near the surface, as before. For a value of $2,000^{\circ} \mathrm{C}$., there is no initial rise near the surface.

In conclusion, two points stand out from the investigation so far carried out. First, all the models of the Earth studied indicate that the temperature at depth has continued to increase throughout geological time, the temperature at the core boundary having increased most probably by about $300^{\circ} \mathrm{C}$. Secondly, although radioactivity plays a major part in the thermal history of the Earth, the initial distribution of temperature dominates thermal conditions at depth and near the surface for the first thousand million years or so. The cosmological questions involved in the origin of the Earth cannot unfortunately be shelved. The discovery of radioactivity, although helping to solve many geophysical problems, cannot in itself wholly explain the Earth's thermal regime.

${ }^{1}$ Jacobs, J. A., and Allan, 1. W., Trans. Moy. Soc. Can., 48, Ser. III, Sect. 4, 33 (1954). Jacobs, J. A., Proc. Assoc. Seism., I.U.G.G. Xth Assembly (in the press).

"Jacobs, J. A., "Handbuch der Physik", 47, Chapter 16 (SpringerVerlag, new edition, in the press).

${ }^{3}$ Bullard, E. C., "The Solar System", ed. G. P. Kuiper, 2, Chapter 3 (Chicago Ưniversity Press, 1954).

- Uffen, R. J., Trans. Amer. Geophys. Union, 33, 893 (1952).

5 Jacobs, J. A., Nature, 173, 258 (1954).

'Russell, R. D., and Allan, D. W., Mon. Not. Roy. Astro. Soc., Geophys. Supp. (in the press).

7 Urey, H. C., Proc. U.S. Nat. Acad. Sci., 41, 127 (1955).

${ }^{*}$ Urry, W. D., Trans. Amer. Geophys. Union, 30, 171 (1949).

\title{
PRODUCTION AND UTILIZATION OF ELECTROMAGNETICALLY ENRICHED ISOTOPES
}

$\mathrm{D}$ URING September 13-16 a conforence on "The Production and Utilization of Electromagnetically Enriched Isotopes" was held in the Cockcroft Hall at the Atomic Energy Research Establishment, Harwell. This was the first meeting to have been arranged on the subject since details were declassified of the calutrons which are now used for enrichment of the isotopes of all the elements. The meeting was attended by Dr. C. P. Keim, Dr. C. E. Normand, and Mr. Boyd Weaver (Stable Isotope Division, Oak Ridge National Laboratory, United States); representatives of separator groups at Copenhagen, Amsterdam, Paris (Commissariat à l'Énergie Atomique, Saclay), Stockholm, Upsala and Gothenburg; members of interested groups in Egypt, Yugoslavia, Belgium and Western Germany; representatives of the nuclear physies dopartments of a number of universities in Great Britain; members of the group concerned with electromagnetic separation at Har. well; and other interested research workers. An unusually lively meeting resulted. The programme covered tho following fields: ion sources, collector problems, chemical aspects and target preparation, utilization, analysis of isotopic abundance, tho design of eloctromagnetic separators, and the use of the separator for active materials as a tool in nuclear research.
The conference was particularly concerned with the stable isotopes other than those of the elements hydrogen, carbon, nitrogen and oxygen, which are enriched by other methods and the application of which has been abundantly dealt with in a variety of reviews. Mr. D. W. Fry, chief physicist of the Atomic Energy Research Establishment, welcomed the delegates and briefly reviewed the twenty-eight papers to be given.

\section{Ion Sources}

At the session on ion sources, held under the chairmanship of Dr. P. C. Thonemann (Harwell), the first paper was by Dr. Normand, of the Oak Ridge separator project, who described tho ion sourcos used in the calutrons. At first the ion source at Oak Ridge was heated by resistance-type (calrod) heaters embedded in the cast-copper charge oven and arcchamber support block. The service temperaturelimit for heaters of this type restricted the operating temperature to about $700^{\circ} \mathrm{C}$. or less, even when the casting of the charge oven was surrounded by stain. less-steel heat shielding. The substitution of graphite components for the copper source castings, and of graphite strip heaters for the embedded calrod heaters, resulted in a source capable of operating at 
regulated temperatures up to approximately $1,000^{\circ} \mathrm{C}$. In order to vaporize metals of the platinum and palladium groups directly into the ionizing arc, a third type of source was developed in which heating was by means of high-energy electron bombardment.

Prof. J. Kistemaker then described the ion sources of the Amsterdam separator, which is a $180^{\circ}$ machine having an ion output of up to $10 \mathrm{~m}$.amp. In particular, he gave a description of the five types of breakdown of the high voltage used in the acceleration of the positive ions from the ion source. Mr. O. E. G. Almén described developments of ion sources in Scandinavia. At present the magnetic type is used exclusively, with the magnetic field either parallel with, or perpendicular to, the direction of the extraeted ion beam. A feature of the Copenhagen source is the use of a vacuum lock for the introduction of the source material so that separation can be made with very short-lived isotopes.

Great interest was aroused by the description, by Dr. R. Bernas (Paris), of a new sputtering-type ion source suitable for the elements of low vapour pressure. In this method the element is sputtered into the arc chamber using a primary argon discharge. This source is still under development, but is extremely versatile and has valuable possibilities for the separation of radioactive elements produced, for example, by bombarding a foil in an accelerator. Dr. R. H. Dawton then reviewed experimental work at Harwell during the past few years on a number of ion sources, including filament sources, radiofrequency types, a sputtering type, a Kunsman source, and sources depending on thermal ionization. Some of these sources have been realized in practice and are being used with the new sector separator recently constructed at Harwell.

\section{Collector Problems}

For the second session, on collector problems, Dr. J. Koch (Copenhagen) was in the chair, and the proceedings opened with a description by Dr. Normand of the unit used for collecting heavy ion beams at Oak Ridge. These beams may amount to more than $100 \mathrm{~m}$.amp. at $30 \mathrm{kV}$., and the difficulties were concerned with the space available as well as the problems of dealing with the energy dissipation and collector erosion. The special constructions which are used to minimize losses by evaporation and sputtering were described and also the arrangements used to prevent cross-contamination of isotopes. A more detailed description was given of the refrigera. tion system necessary for mercury collection, and of the special collector arrangements. Dr. M. L. Smith then dealt with experience at Harwell in the samo problem, discussing particularly the chemical contamination of isotopes. He also described arrangements suitable for the collection of the very volatile elements such as sulphur and chlorine, the gases oxygen and nitrogen, and the rare gases.

The problem of the collection of mercury was returned to by Dr. Ranc (Saclay atomic energy group), in whose system the source is supplied with mercury vapour while collection is achjeved on gold foil cooled with liquid nitrogen. To obviate contamination from the mercury vapour existing in the tank at room temperature, a secondary liquid-air trap is placed near the source and provided with a slit, so that only the directed mercury ions can pass.

\section{Chemical Aspects and Target Preparation}

In the session on chemical aspects and target preparation, under the chairmanship of Mr. Boyd Weaver (chief chemist of the Oalk Ridge Stable Isotope Group), Dr. K. O. Neilsen (Copenhagen) considered the penetration of ions into solid lattices. This problem is important in connexion with the preparation of gas targets and is, also of interest to several other studies such as that of the recoil atoms from nuclear reactions and, in particular, the field of radiation damage. The theoretical conclusions obtained from his work were compared with experimental studies by nuclear reactions which enable the distribution in depth to which the ions have penetrated to be found.

Other methods of preparing separated isotopic targets for nuclear reaction studies were described in the next paper by Dr. Smith. He commented on the advantages of a central station for isotopic target preparation, considered the requirements for nuclear targets and described some typical techniques. The most versatile is vacuum evaporation, which besides its application to salts can also be used for evaporation of the refractory metals such as magnesium and silicon to give elemental deposits by using tantalum filaments as a source on which the oxide is reduced. This method can also be used for the preparation of metallic targets of the alkali and alkaline-earth metals, and special handling techniques were described by Dr. Smith for these. Direct deposition in the separator is used for various gases and for boron, which stays down with less than 10 per cent sputtering on a variety of metallic backings.

Mr. Boyd Weaver then described in some detail chemical aspects of the work on electromagnetic separation, dealing first with the preparation of charge materials. So far, more than fifty elements have been separated, and in most cases some special chemical operations have been necessary or helpful in the supplying of suitable charge materials for the separators. Whenever possible, a variety of forms of each element has been prepared in order to make comparisons between halides, oxides and elements. Improvements in operating efficiency have been attained by applications of such techniques as vacuum dehydration and pelletizing. Half the elements still unseparated consist of the rarer rare earths. In order to make as much as a kilogram of each of these available, various methods of separation have been explored. Separation by continuous counter-current extraction between nitric acid and tributyl phosphate has been developed and applied to the preparation of unusual quantities of these elements.

Turning to the processing of the enriched material in the collectors, Mr. Weaver pointed out that these are always contaminated by a large number of other elements which have to be removed in order to obtain the pure material needed for nuclear research. The purifications require an unusual type of quantitative analytical chemistry, in which the emphasis is on separation rather than on measurement of quantities. Purification schemes suitable for quantities from milligrams to grams have been developed for each of the elements.

\section{Applications of Electromagnetically Enriched Isotopes}

In this session the chairman, Dr. Keim, gave a wide-ranging review of the achievements of the stable- 
isotopes programme in the United States, which, so far, has involvod the dispatch of more than 2,600 consignments of stable isotopes to users in sponsored establishments of the Atomic Energy Commission, government agencies, universities and industries. Dr. Keim pointed out, however, that the contributions which stable isotopes are making to basic science and in a growing number of practical applications are not widely appreciated. This is largely because stable isotopes are at present of greatest value to the research physicist in fundamental investigations which receive little publicity. There is also a reluctance to use enriched samples, as they appear to be expensive, although, in fact, only small quantities may be needed, while other savings may justify their use. They are, too, more difficult to use than radioisotopes, but improvements in massspectrometry techniques will help here.

Di. Keim quoted the immense range of work already published and went on to describe a few particular applications of stable isotopes. These included: their use as starting materials in the assignment of masses to artificially produced radioisotopes and in investigations of their disintegrations; the determination of neutron absorption, scattering, and total cross-sections at all neutron energies; atomic spectroscopy, especially in the study of hyperfine spectra, isotope shifts, nuclear spins, etc. ; the determination of nuclear energy-levels, and studies of isomerism; superconductivity, selfdiffusion, coefficients of expansion and other solidphysics studies; $\beta$-spectroscopy using enriched isotopes like potassium-40 and beryllium-10 ; use for pile and cyclotron bombardments to produce radioisotopes of high purity and high specific activity, and to produce certain isotopes otherwise unavailable at a reasonable cost; use as tracers in isotope-dilution analysis, a technique which is now being increasingly applied in geology, agriculture, metallurgy and biology.

Dr. W. D. Allen (Nuclear Physics Division, Harwell) then described the investigations which have been carried out with materials supplied by the separator group at Harwell. He illustrated his lecture by details of a few particular investigations out of many which have been carried out during the past five years using the six hundred and fifty consignments which have so far been dispatched by Harwell to users in many countries. Dr. Allen first described reaction studies with the van de Graaff and Cockcroft-Walton machines and then gave a detailed review of a variety of studies using neutrons, describing, in particular, the current work on fastneutron capture in the Zephyr reactor. This work refines the earlier studies by D. J. Hughes of the variation of cross-section with atomic weight which has some cosmological implications and also throws more light on the magic-number nuclei.

Dr. Allen considered a variety of other topics, including $\beta$-ray studies; $(n, p)$ reactions using $14-\mathrm{MeV}$. neutrons; several othor reaction studies; and photo-disintegration. In a forecast of future work with electromagnetically enriched isotopes he said that the work on neutron cross-sections in all energy-ranges is only just beginning. Studies in Coulomb excitation show much promise; the work with $1-\mathrm{MeV}$. high-tension sets in many universities will continue and will gradually extend to the masses in the range oxygen-16 to calcium-40. He also mentioned tritium and-helium-3 reactions and the very interesting subject of double $\beta$-decay.
Dr. J. C. Kluyver (University of Utrecht) then described the investigations he has been making on $(p, \gamma)$ reactions using enriched isotopic targets of magnesium and silicon. It is imperative to use enriched targets for this study in order to assign correctly each proton-capture resonance. With $a_{0}$ non-separated target, this assignment is very difficult as aluminium-25 and -26 have nearly equal half-lives, and the long-lived aluminium-26 may easily be confused with the stable aluminium-27 as product nucleus. As natural magnesium contains 79, 10 and 11 per cent of the isotopes of weight 24,25 and 26 , respectively, isotopic targets of magnesium-25 and -26 are nearly tenfold enriched, and a corresponding gain in the $\gamma$-ray yield is obtained. Also, the analysis of the weak ${ }^{25} \mathrm{Mg}(p, \gamma)$ resonances is greatly facilitated by the reduction in the intensities of the fortytimes stronger ${ }^{26} \mathrm{Mg}(p, \gamma)$ resonances in their neighbourhood. From this work the energy-level schemes of aluminium-26 have been worked out. The use of isotopic targets of other elements in $(p, \gamma)$ reactions will have similar advantages.

Dr. H. G. Kuhn (University of Oxford) then gave a polished account of applications in spectroscopy and in solid physics. He said that the differences in the masses of isotopes give rise to vibrational and rotational isotope effects in molecular spectra. Their study, either by optical or microwave methods, can be an important aid in the analysis of spectra. In atomic spectra the mass effect is predominant in the lighter elements, and its study is necessary for isolating the more important 'volume effects'.

A great variety of interesting spectroscopic effects are caused by differences, between the different isotopes, of their values of nuclear spin (I), magnetic moment $(\mu)$, electric quadrupole moment $(Q)$ and distribution of charge inside the nucleus. Investigations of these effects are often very difficult, and sometimes impossible, unless highly enriched isotope samples are available. Light sources containing certain pure or highly enriched isotopes can produce very highly monochromatic light. This property makes them suitable for use as wave-length standards, for interferometric measurements of gauges of great length and for purposes of adjustment of interferometers of long path.

Most properties of solids depend on the atomic masses, and the study of isotope effects can be a powerful means of testing the validity of theories. Such effects are usually small and difficult to measure, but some variable results on the transition points of supraconductivity and on the rate of change of electrical resistance of metals with temperature have already been obtained. Apart from their bearing on the theories of conduction and supraconductivity, results of this kind are likely to contribute generally to the knowledge of the structure of solids.

\section{Isotopic Abundance Analysis}

The session on isotopic abundance analysis, with Prof. J. H. E. Mattauch (Max-Planck Institut für Chemie, Mainz) in the chair, was one of the most vigorous of the conference. It opened with an account by Dr. Keim of work at Oak Ridge, where the mass-spectrometry section is concerned with analysis of samples from the separator group and also with a variety of research problems in collaboration with many institutions. During the past few years the tendency has been to move to techniques for the less-volatile elements requiring high source-temperatures, and so special furnace 
and thermal ionization methods have been especially developed. Techniques of small-sample loading employing micro-pipettes and a microscope were described, and the advantages and problems of working with extremely small samples of a few micrograms were discussed. Details were given of a range of instruments developed in the group, including one made by the General Electric Corporation. Mr. G. H. Palmer then described the work at Harwell in this field. He said that, as in Oak Ridge, the emphasis has been on handling solid samples rather than gases, and described the precautions necessary for this kind of work. In particular, he discussed the difficulties of quickly comparing samples and obtaining ion beams of constant intensity. He then described a new mass spectrometer which has been developed at Harwell, in association with Metropolitan.Vickers Co., Ltd., especially for solid analysis and which incorporates both furnace and triple-filament assembly types of sources together with a variety of detection devices. In a selective review of Harwell work, he described results obtained with lithium, lead and uranium. Mr. Palmer then went on to read a paper by Prof. G. D. L. Schreiner and Dr. R. T. Jamieson (Johannesburg) on age determination by isotopic-dilution techniques. This was a valuable and detailed discussion of the theoretical basis and the errors in the technique, with particular emphasis on the rubidium-strontium mothod.

Among the papers at this session was a notable contribution by Dr. H. Hinterberger (Mainz). He first dealt with the principles and mass-spectrometric techniques used in isotopic-dilution methods, and then went on to survey work in this field. After a discussion of the potassium-argon method used in the determination of the age of the Earth, he deseribed his own recent contribution with the newly developed rhenium-osmium method. This very interesting work is probably a unique example of the success of mass spectrometry over the counting methods.

The last paper of the session, by Dr. A. H. Gillieson (Chemistry Division, Harwell), discussed methods other than mass spectrometry by which measurements may be made of isotopic abundance. The methods dealt with can be briefly listed as : neutron source methods (neutron absorption, radioactivation); optical methods (emission spectroscopy, absorption spectroscopy, refractive index); and mass methods (density). Evidently there are several alternatives to mass spectrometry which should be considered in practical problems.

\section{Design of Electromagnetic Separators}

This session, with Prof. Kistemaker in the chair, brought excellent papers from three leading workers in the field, the first being Dr. Normand, who gave a detailed and beautifully illustrated review of the Oak Ridge piant with technical details of the installation. The present equipment includes two small and two large calutrons which are operated for seven days a week on three shifts, and it is being planned to extend the facilities by adding two more large calutrons shortly.

Dr. Dawton then discussed the design considerations which led to the construction of the new highresolution separator at Harwell. This machine, which is intended for enrichment of the isotopes of the transuranic elements and of fission products where high activities may be encountered, is a 4 -ft. radius, $90^{\circ}$ separator of the Nier type and is as fully automatic as possible. A detailed description was given of the remote-handling device for removal of the collectors containing the enriched product without exposure to air. Recent experimental results on the properties of plutonium of particular importance to its electromagnetic separation were also described.

Dr. Koch gave a masterly review of the Scandinavian separators in which, speaking for the groups in the three Swedish laboratories as well as for his own laboratory at Copenhagen, he described all four instruments. These separators have much in common, and a best basic design for use in nuclear studies has clearly been developed by these workers during the past fifteen years. The application to research on short-lived isotopes by integrating the separator with other tools such as the cyclotron and $\beta$-ray spectrometer to make a unified assembly which can attack most powerfully nuclear problems of all types was demonstrated. Dr. Koch concluded by making a plea for the preparation of the widest variety of stable and radioactive isotopes in the state of highest purity.

Two papers were presented to the conference at the last moment, and these fitted best with the session on design. The first was a theoretical paper by Dr. H. Bruck (Saclay), who showed that, for a magnetic separator of the usual sector type, the use. of the alternating gradient principle in magnet design instead of the usual arrangement of a uniform magnetic field so far employed gives greater dispersion. In effect, one can have higher dispersion for the same radius, or the same dispersion for a smaller radius. The last paper was a possibly prophetic contribution beautifully presented by Dr. O. Osberghaus on the use of alternating-gradient electrical focusing for mass separation. In this system, using an arrangement very similar to that used on high-energy machines for beam ducting, a direct-current and radio-frequency voltage are applied to alternate plates. An experimental unit using this system has been successfully run at Bonn, and a larger one is now being constructed. This device is limited to small ion beams because of the lack of space-charge stabilization, but it seems that the principle may be useful for beams of up to $100 \mu$ amp. Dr. Osberghaus, who is working with Prof. W. Paul at Bonn, envisages two systems, one in which a selected mass is passed alone and the other in which a selected mass can be rejected.

\section{Separation of Active Material}

The last session, under the chairmanship of Dr. Bernas, consisted of two papers, though the earlier paper by Dr. Koch had, in fact, largely dealt with the same subject. Dr. G. Andersson reviewed nuclear studies in Scandinavia carried out with electromagnetically enriched radioisotopes. He described first the use of the separator for the preparation of samples of known masses for beta-ray spectroscopy and, in particular, for work on the rare-gas isotopes which are studied after trapping in foils. Extensive work on the radioactive nuclides of krypton and xenon has been done at Stockholm by this technique.

Dr. Bernas then dealt with his work at Saclay, where he has constructed a medium-sized separator particularly suitable for the elements of light and medium mass. This instrument can separate tens of milligrams of a given element in a day, while being sufficiently flexible to separate in rapid succession 
the radioactive isotopes of widely differing elements. Actual experience with this instrument has shown that both modes of operation are possible and successful; they provide alternative experimental approaches to the particular problems, and Dr. Bernas discussed their relative merits. He also described in some detail the work on technetium, which is propared by cyclotron irradiation of a molybdenum target and thon mass-separated. An interesting example of the application of the separator to alpha-particle spectroscopy was also described.

A lively discussion followed every paper, and it was felt that the conference had had some important results in directing attention to a great variety of applications of mass spectroscopy, and in describing many applications of electromagnetically enriched isotopes. Hitherto, the more spectacular application of radioisotopes and the relative ease of their techniques of use has overshadowed the uses of stablo isotopes. It was felt that the present conference would show the rich field of work still waiting to be done in the latter field. In summing up, one might say that three main types of activity could be clearly recognized at the conference : the work of the two groups at Oak Ridge and Harwell in the provision of enriched isotopes for basic research of all kinds; the work of several European groups in the application of medium-sized isotope mass-separators to the direct study of a great range of nuclear problems; and lastly the large numbers of research workers who essentially are applying the enriched isotopes provided by Oak Ridge and Harwell to a great variety of researches. An important result of the conference was that the common interests of these groups were clearly recognized and great stimulus given to their work. It is hoped that on publication of the proceedings a much fuller appreciation of this new field will be generally gained. The proceedings, including lectures, discussion and illustrations, will be published shortly by Butterworths Scientific Publications, Ltd., London.
M. L. SMrry

\section{MANAGEMENT AND HUMAN RELATIONS}

\begin{abstract}
$\mathrm{A}^{\mathrm{N}}$ $\mathrm{N}$ attendance of nine hundred members at the annual conference of the Institute of Personnel Management at Harrogate during October 7-9 is an indication of the growing importance attached to the personnel function of management in British industry. Those attending came from very large and small manufacturing firms, departmontal stores, nationalized industries, publie corporations, insurance and other commercial concerns, educational institutions and the Armed Forces. A number came from overseas. At the plenary sessions speeches of a high order were given, and at the various sectional meetings the investigations now being made into the behaviour of people in industry by a number of research organizations woro discussed.

Sir John Hunt, leader of the British Mount Everest Expedition in 1953, opened the conference with a challenging paper on the needs of youth in a Welfare State. There are, he said, many signs that the spirit of young people is either flagging or misdirected; there is too much apathy and hooliganism abroad. A great deal of fine work is still being done to help young people find their feet and set them on the road to good citizenship, and yet it would be foolish to be complacent and suppose that more could not and should not be done. Something is lacking in the opportunities offered, in the guidance given: What is this lack? First, the incentive to achievement is generally inadequate. If it is accepted that one of the needs in every individual is a sense of purpose, then the opportunity of achievement is an essential step in providing that purpose. Secondly, there is a great need for leaders, both in numbers and in quality. The paid official alone will not do, and the need is for more volunteers, because they bring to young people a variety of experience of contemporary life. Ideally, leaders should have graduated from a similar background to members of the group - the best leadership is leadership from within the group, not from above it. The main source of youth leaders should be ex-members of the organizations themselves rather than older men implanted from outside and from a different environment.
\end{abstract}

A scheme should be devised which embodies incentives and sets certain targets or standards of achievement. How can such a scheme be made attractive and how can it work? Can a scheme of wide application be devised which might draw in more young people? Sir John suggested that it should be planned under four headings: the development of a hobby-a technical, scientific or artistic skill ; the development of physical fitness-this calls for moral as well as physical stamina; outings, whether on foot or cycle or in a small boat-these are of tremendous value to the character training of young people and can be extended progressively until they assume the character of expeditions; and the instilling of the spirit of service-this is best caught by example, and the example must come from the leader.

Sir John was followed by Sir Richard Coppock, general secretary of the National Federation of Building Trade Operatives, who said that, in the main, the weakness of the building industry is on the managerial plane. He was particularly concerned about the missed opportunities for consultation between management and worker. The experience the workers possess is the basis of Britain's prosperity and is unequalled in any board-room in the country. Things do not go wrong on the drawingboard, he maintained, but when machines are being run and built, and he quoted the Comet aircraft as a good example of this. Half the difficulties in his industry arise because the employer does not enlist the goodwill of his men by telling them all the facts. The great problem facing management is how to handle men. One of the most vicious things they can do, when an unofficial strike takes place over some potty tyranny, is to say: "You must all go back at once. We cannot see you". This, said Sir Richard, puts the trade-union official in a most humiliating position-he is humiliated before negotiations have even begun.

Some management problems in the British Broad. casting Corporation were discussed by the directorgeneral, Sir Ian Jacob. The Corporation employs 